\title{
MANAGEMENT STRATEGIES ON DIGITALIZATION IN THE HEALTH FIELD
}

\author{
Vasile APOSTOL ${ }^{a^{*}}$ \\ Bucharest University of Economic Studies, Romania
}

\begin{abstract}
This article presents the analysis of the current state of digitalization in the public health field, more precisely in school medicine in Bucharest, the impact and consequences for the daily activity of medical staff on one hand and to the decisions maker on the other hand and also the steps undertaken to optimize the process by digitalizing the medical assistance in public schools in Bucharest. This analysis was the starting point for an active involvement in the digitization of school medicine practices and implicitly their alignment with European standards.

The purpose of this article is to highlight a macro-level management perspective in the digital era. Digital evolution is absolutely necessary and natural in adapting the system to current requirements, the need to have access to important health data at all times being for the benefit of all stakeholders. Another important factor that led to the digitalization of the school medicine network was the crisis generated by the COVID-19 pandemic, which taught us to adapt and move from lost hours for a medical consultation and paper prescriptions to online consultations.

Analyzing the imposed situation, we chose to take a major step towards progress, capitalizing on the time of medical staff and patients and the time at management level to properly adapt and implement medium and long term decisions.
\end{abstract}

KEYWORDS: digitalization, digital era, health care, management, school medicine

\section{INTRODUCTION}

Digitalization in healthcare is a set of tools and services that use information and communication technologies (ICT) and have benefits for both patients and consumers, as well as for healthcare professionals and the healthcare system as a whole. Digitization not only provides increased access to information that can help maintain and improve health, but also facilitates increasing the accountability of all parties involved in this process. Moreover, digitization allows greater connectivity and a constant exchange of information, with major benefits in research, faster diagnosis, prescribing personalized treatments and better results, both medically and in terms of cost efficiency and improved access to quality health care.

On March 30, 2020, the Government of Romania approved the Emergency Ordinance no. 38 on the use of electronic documents at the level of public authorities and institutions, which allows the use of electronic documents both for communication in digital format between state authorities and institutions and between them and individuals and legal entities, and ensuring the development of vital processes of the state by implementing computer programs and means based on information and communication technology.

Thus, once again, right at the beginning of the SARS-CoV-2 pandemic in Romania, the need for public institutions to digitize and thus allow citizens to interact with them in the online environment was strongly emphasized.

\footnotetext{
*Coresponding author. E-mail address:vasile_apostol@yahoo.com
} 
The digitization of the public sector should not be considered solely a technical project or a project merely about improving efficiency, freeing up resources and modernizing service delivery. Instead, digitization should be seen as a substantial reform driven by strong programmatic ideas and ideals and an often-unquestioned digitization imperative. (Plesner, U., Justesen, L., \& Glerup, C. (2018). Digitalization in health has been a priority of the Bucharest City Hall in recent years, offering an extraordinary opportunity to put technology at the service of healthcare. The steps have been finalized so far with the elaboration of some programs / projects in this field, some of them currently being implemented in health care.

One of the large-scale projects developed in Bucharest is the digitization of the school medicine network, 609 school medicine offices, a management tool necessary for creating a unitary way of working and establishing public health policies and standards among schoolchildren.

The objectives of the digitization project were ambitious and complex, but absolutely necessary in relation to the current need imposed by severe allergies among pupils, medical peculiarities and current pandemic context resulting in a more efficient management of children's and young people's health.

The project is unique at the national level in terms of scale and complexity model of good practices for other local public authorities in the country.

\section{DATA ANALYSIS AND RESEARCH METHODS}

The approach of the topic of this article was based on a series of techniques and tools for investigating and documenting the phenomena and processes, on one hand, within the school medicine offices and, on the other hand, in the field of digitization.

The complexity of the information obtained and the processes led to the use of mixed methodologies, so that the integration of the results leads to the achievement of the established objective.

As a systematic process of collection and analysis of the obtained data, the scientific method was used, in the first phase.

The steps of the research process applied in this research are presented in figure 1.

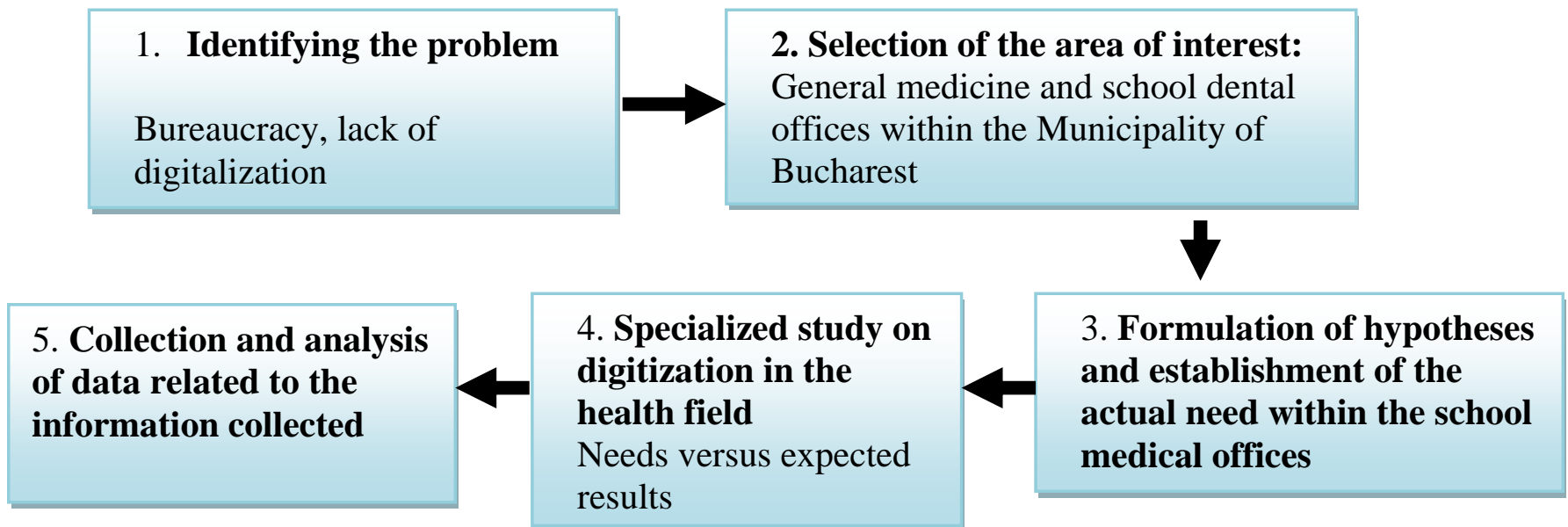

Figure 1. Research Process Diagram

Source: Created by the author

Figure 1 underlines the complexity of the process, the flow towards the analyze, critically needed to collect all the information for the management decisions. 
The analysis of the existing working process and the definition of the requirements of the new system represented the first stage of the life cycle of the digitalization system development within the school medicine offices process.

The study of the working process, attributions and inter-institutional and intra-institutional communication included activities that aimed at knowing the responsibilities and connectivity both as macro and micro level and knowing the shortcomings and restrictions of the current way of working to the actual needs of the offices of school medicine.

The method used in the analysis of the information system requirements was the interview, using the JAD methodology.

The interview is a very common method for gathering information from the working flow system. The interviews conducted within the institution, allowed the team responsible for analyzing the data and providing the solution, modeling them according to the specific objectives of defining the functionalities of the expected information system.

The employees able to provide the information closest to reality were interviewed, both from the school medicine offices and from internal structures that are working in direct interaction. Another step made in this research method was to collect the correct information that required the interview of both the management staff and the executive staff, the competence of the interviewed subjects being previously verified. Both steps have been used to highlight the constraints and implications of the vertical and horizontal communications flow.

The main idea of using the JAD method was to bring together all those interested in the development of the system: key users (medical staff), internal structures directly involved in the interaction with healthcare professionals. From this point of view, JAD is similar to the group-level interview. However, in the JAD session a certain sequence of activities was followed, based on well-established roles.

Prototyping is an interactive process by which the team responsible for analyzing data and providing the solution and users discussed a rudimentary version of an information system, a version that for about a year has undergone changes and updates, depending on user feedback.

Interviews were used to gather information about user requirements, but by prototyping, the operation could be simplified.

Prototyping was used in the analysis of the requirements for the implementation of digitization in school medicine offices for the following reasons:

- the user's requirements were not very clearly formulated or well understood;

- certain means of work are used (predefined forms and reports).

\section{SOURCES OF FUNDING}

From the research process, I identified that a key issue in the implementation of digitization, is financing, for long-term digitization process, and one important source of money can be to attract financial funding from external programs.

Accessing and attracting financing funds, with the purpose of generating progress and prosperity, represents an essential management function necessary for the development at national and local level, overcoming the financial deficit related to public institutions and establishing new premises for carrying out the strategic plan.

Thus, in the critical sectors and implicitly in the sanitary field, the following were identified in addition to funding sources:

\subsection{National recovery and resilience plan}

Romania will have at its disposal 29.2 billion Euros through the Recovery and Resilience Mechanism for the implementation of the National Recovery and Resilience Plan (PNRR). 
In the field of digitization, the proposed reforms are aimed at digitizing government data registers, interconnecting and interoperability of existing IT systems, promoting technologies that increase the quality of life and updating digital services and launching new ones.

The main proposed health reforms encompass organizing the public e-health service, including telemedicine (by equipping it with IT equipment / devices) and software necessary for the digitization of the current activity of the health units also improving the quality and accessibility of medical services for critically ill patients and making specific investments, and increasing the resilience of the public health system by making specific investments necessary for the regional storage of medical equipment in crisis situations.

The digitalization of public health services is one of the major areas of investment, along with investments in modernizations, relocations, construction of new health facilities, or investments in oncology, cardiology or the construction of warehouses for medical equipment and medicines.

\subsection{Health operational program $2021-2027$}

The estimated budget of the program is over 4 billion Euros (ERDF + ESF + National ContributionState Budget), for the programming period 2021-2027.

The strategic vision of the operational program: "A nation with healthy and productive people through access to quality preventive, emergency, curative and rehabilitation services."

\section{Among the investment priorities that will be financed within the Health Operational Program 2021-2027:}

- The infrastructure of the regional hospitals Cluj, Iași and Craiova - the second stage, as well as investments in the infrastructure of the hospitals with major territorial impact;

- Primary and community health care services and services offered on an outpatient basis: infrastructure of family doctors' offices, infrastructure for school and community health care services, including dental care services, health units providing outpatient health care, screening centers;

- Rehabilitation, palliative and long-term care services, including investments in systemic measures and for the development of staff skills, as well as medical services for vulnerable people;

- Increasing the efficiency of the medical sector through investments in infrastructure and services, by developing: the transport system, taking over, as well as units dedicated to the care of critical patients, including children, pediatric neurology-rare diseases, national transfusion system, network of regional laboratories, hospitals the medical genetics network;

- At the same time, the following will be financed:

O Implementation of population screening programs / diagnosis and treatment of early stages especially for people from vulnerable groups, by segments that are the main causes of mortality: cervical cancer, breast cancer, colorectal cancer, hepatitis / HIV AIDS, metabolic screening, tuberculosis, genetic testing, screening of common risk factors for chronic diseases, etc.

- Measures for early diagnosis and / or antenatal / neonatal / postnatal treatment.

- Implementation of early diagnosis and treatment programs, especially for people belonging to vulnerable groups such as lung cancer, prostate cancer, gynecological cancers, head and neck cancers, genetic testing, etc.

- An important component for which European funds will be allocated is the one that aims to control the infections associated with the medical act.

- Innovative approaches in medical research. Investments in research will support:

- Infrastructure development, modernization, resumption of vaccine production and development of new pharmaceutical products in the National Institute for Medical-Military ResearchDevelopment "Cantacuzino";

○ Realization of a research-development infrastructure in the field of genomics;

- Strengthening research and development capacity in the field of cancer treatment. 
- Digitization of the medical system: Investments will support:

○ Restoration and modernization of the computer system of the National Health Insurance House;

○ Development of the National Observatory for Health Data;

o Internal and external digitization of medical institutions as well as standardization of information flows.

- Promoting modern methods of investigation, intervention, treatment - development of centers of excellence in innovative cell therapies in hematology and bone marrow transplantation, digestive diseases and liver transplantation, urology and kidney transplantation.

\section{DESCRIPTION OF SCHOOL MEDICINE IN BUCHAREST}

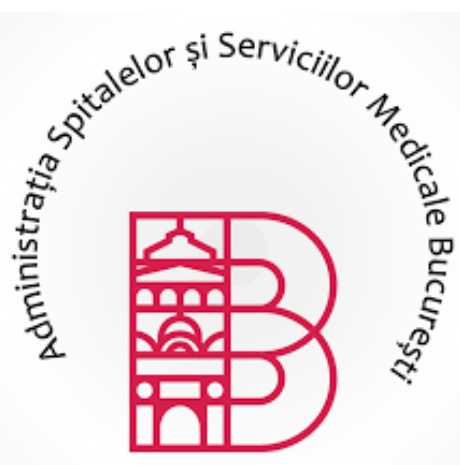

The Bucharest Hospitals and Medical Services Administration (A.S.S.M.B.), a local public institution, was established in 2008, and is organized and operates based on the Administrative Code, Emergency Ordinance no. 57/2019, O.U.G. no. 162/2008 regarding the transfer of the set of attributions and competences exercised by the Ministry of Health to the local public administration authorities, with the subsequent modifications and completions and the provisions of the Government Decision no. 56/2009 for the approval of the Methodological Norms for the application of the Government Emergency Ordinance no. 162/2008, with subsequent amendments and completions, as well as of the H.C.G.M.B. and Provisions of the Mayor General (https://assmb.ro/page/baza-legal).

\section{Image 1. - ASSMB logo (Source: ASSMB website)}

The main purpose of the management process is to change the paradigm in approaching the health of the capital's citizens, corresponding to the European landmarks of the third millennium, through the decentralized administration of medical care units (hospitals) in the city. The main activity of ASSMB consists in the financing, but also the administrative coordination of the hospitals at the level of the local authority.

The Network of School and University Medicine in Bucharest, administered by the General City Hall of Bucharest through the Bucharest Hospitals and Medical Services Administration (A.S.S.M.B.), has 452 general medicine offices and 157 dental medicine offices in kindergartens, schools and universities.

Medical assistance is provided by 265 doctors (119 general medicine practitioners and 146 dentists) and 602 nurses (475 general medicine practitioners and 127 dental assistants), for a number of approximately 250,000 pupils and 121,000 preschoolers and students, according to the provisions of the Order issued by the Ministry of Health and the Ministry of Education and Research, no. 438 / 4.629 / 2021 on ensuring the medical assistance of preschoolers, pupils in pre-university education units and students in higher education institutions for maintaining the health of communities and for promoting a healthy lifestyle.

The medical staff within the school and university offices provides medical assistance to all preschoolers, pupils and students from the state pre-university and university education units in Bucharest.

The school medical and dental assistance services are composed as follows:

a) services for ensuring a healthy environment by identifying and managing the risks for the health of the community, ensuring the functional circuits (space and tools), verifying the observance of the public health regulations;

b) prophylactic services for maintaining individual and collective health: 
(i) performing daily epidemiological triage in preschools, before entering the community;

(ii) performing epidemiological triage in pre-university education units, after school holidays and whenever necessary;

(iii) carrying out immunizations in special epidemiological situations, established by order of the Minister of Health, in compliance with the law on patients' rights;

(iv) initiating measures to control and control communicable diseases, as appropriate;

c) health surveillance and assessment services, including oral health, early detection of diseases of the dento-maxillary apparatus, early detection of chronic or chronic diseases, monitoring of patients with chronic diseases, issuance of necessary medical documents;

d) free curative medical actions: medical services to ensure the state of health, including oral health, granting medical and dental consultations, issuing referral tickets to doctors of other specialties, issuing free prescriptions and providing first aid in case emergency;

e) health education activities, including reproductive health, oral health, and the promotion of a healthy lifestyle;

f) counseling for the early application of curative and medical recovery treatments (according to Ministry of Health and the Ministry of Education and Research - 2021).

\section{DIGITALIZATION OF THE SCHOOL MEDICINE NETWORK}

The digitization of the school medicine network represented an extensive management process, carried out over a period of approximately one year (2020-2021), correlated with the need for digitization and the current pandemic context, the end result being the standardization of public health policy at the level of services for school medical care and dentistry, being one of the most elaborate projects for the digitalization of medical activity carried out in Romania, even in the conditions in which the fight against the COVID-19 pandemic has concentrated, in the last year and a half, almost all efforts and resources at the national level.

The medical and administrative staff from the school medical offices previously carried out their activity based on the work procedures elaborated at the level of the institution. The manipulated information was processed manually using typed or improvised forms, the data being available only on paper. Despite human efforts, the activity took place in time intervals appreciated by the beneficiaries of the services as being unsuitable for the technological conditions currently available. The "paper" mode of operation produces negative effects for:

$>$ patients: as the staff's attention is directed very much outside the actual medical act, there are delays or problems in information processing, planning or monitoring; the quality of the medical act is negatively affected by virtue of the manual operation of the information;

$>$ the image of the institution: the services offered to patients cannot be centralized in due time so that the collective measures for the implementation of prevention policies can have the desired efficiency, and the efforts of medical staff in following patients are unjustified in the technological conditions available in the market; the technology exists, and the lack of its implementation in ASSMB is visible;

$>$ the current activity of the medical and administrative staff: response times are particularly long; reports contain errors or inconsistencies; management is forced to make decisions based on subjective considerations with a direct impact on the entire activity.

The introduction of a computer system for the management of medical activity within the school offices proposed results such as immediate increase in the quality of medical services provided to patients, especially by increasing the attention paid to the medical service and the correctness of the information processed by doctors and nurses, increasing the degree of patient satisfaction, quality of treatment for preschoolers, pupils and students, elimination of problems caused by the speed of data 
processing, including reporting to coordinating physicians, the major decrease in time allocated by medical staff for information processing activities or for repetitive non-medical activities has the immediate effect of allocating this time to medical activities or administrative procedures carried out in the interest of patients.

Another expected result is to correct the planning and traceability of the activity, an IT system automates the processes and allows the traceability of activities, and also, modernization, the introduction of an IT system at ASSMB level determines that the entire activity, from the operational one to reporting and statistics can be done on time by the medical offices.

Thus, ASSMB is technologically aligned with an updated way of working in which the medical staff has the necessary resources to pay appropriate attention to the treatment of patients.

Thus, the full digitization of the activity of the 609 school and university medical offices in Bucharest, administered by the Bucharest City Hall, through A.S.S.M.B, was completed on September 1, 2021.The need to digitize school medical offices started from a detailed analysis, which concluded that the processes performed by medical staff have a high degree of difficulty and involve high operational risks, reported with low consequence and particularly process management, complex and difficult to follow.

The need to computerize the medical assistance and dental school medicine offices is objective and its main purpose is to streamline the activity at the level of the institution and the medical offices. Although at the institutional level the staff knows the operational flows, the rules governing the activities and responsibilities specific to the position, as well as to a large extent the connections with the medical offices but also with the institutions with which they are directly related, the communication imposed by the bureaucratic process makes it difficult to carry out specific medical activity. The automation of workflows with the help of an IT system for the management of activities in medical offices considerably reduces the negative effects of manual data processing and orients the current activities of medical staff especially to patients and less for bureaucratic purposes. The computerization of the school cabinets with a software product corresponding to the needs will have positive effects on the way of working as well as on the existing procedures, and the effect will be immediate and of interest for the young patients. For the implementation of such solutions, the medical and administrative staff of the institution provided all the necessary support, so that the implementation of this digitization solution can be implemented efficiently.

The staff has been trained to use the medical software and is already familiar with the interface and how it works, and all general medicine and dentistry offices in state educational institutions in Bucharest are currently equipped with high-performance computers and printers, so the activity can be carried out in optimal conditions.

The digitalization of the school medicine network in Bucharest consisted in providing computers and peripheral devices, on the one hand and implementing high-performance medical software, which provides medical staff with a series of tools that will substantially streamline the work of offices operating in kindergartens, schools and universities in the Capital, as follows:

$\checkmark$ Electronic patient file - accessing the patient's medical history and medical forms;

Quick consultation of radiographs, prescriptions, patient diagnoses - general status, treatment plan, dental status, questionnaires completed by the patient, interactions between the patient and other school medical offices, etc.;

$\checkmark$ Evidence of treatments and consultation of treatment history;

Reports - the realization of situations that contain processed and synthesized data, which provide critical information in the process of developing medium and long term strategies;

Stocks - records of medicines, sanitary materials, fixed assets and consumables allocated per office;

$\checkmark$ Automatic backup - full data security by performing periodic backups in the cloud, at the highest level of security and operational reliability. 


\subsection{The specific objectives of the digitization process}

The digitization of the network of general and dental school medicine in Bucharest has had the following specific objectives:

$>$ Implementation within the medical and dental offices subordinated to ASSMB of a modern electronic platform that facilitates and streamlines the daily activities and ensures the records of patients;

$>$ The standardization of the reporting process within the general medicine and dental offices to ASSMB, which must result in a more efficient management of the available information;

$>$ Reducing the time needed to collect information from medical and dental offices, as well as the time for developing and consolidating integrated reports;

$>$ Ensuring an efficient access to information, as well as increasing the speed of data exchange held by medical and dental offices;

$>$ Ensuring an efficient control of the quality and veracity of the reported information;

$>$ Strengthening the institutional culture of evidence and reporting, which will contribute to increasing the level of data accuracy;

$>$ More efficient use of computer technology in medical and dental offices and reducing the need for the circulation of information on paper;

$>$ Ensuring the transparency of public information regarding the activity of the medical and dental offices subordinated to ASSMB.

$>$ Assimilation of existing information in electronic format in the new application and its integration in an updated computer system that will allow the loading and processing of information in a structured way;

$>$ Ensuring the monitoring of the health status of school and pre-school children, as well as of students from higher education institutions by implementing a database that includes existing information in electronic format (editable) and additional ones and their integration in such a way that real-time reports can be obtained;

$>$ Management of consistent and quality information that aims to increase the quality of the medical act;

$>$ Providing decision support to ASSMB management staff;

$>$ Connecting all medical and dental offices subordinated to ASSMB involved in the medical act of triage, consultation and treatment of children, pupils and students in educational institutions in Bucharest.

\subsection{The functionalities of the medical informatics program}

The application is a web type, the advantages compared to desktop software being already well known. In this regard, I recall the following advantages: the application can be used on a very large scale, runs directly from the browser and is very convenient to use. The main functionalities and the benefits of it for medical staff and patients, basically the benefits of digitalization in this case, as described hereunder, derived from the research process:

Patient electronic file: Each student will have an electronic file, so that the medical staff can introduce the clinical examinations, add the diagnosis and access the patient's medical history and medical forms (quick consultation of radiographs, prescriptions offered, patient diagnoses), general status, treatment plan, dental status, questionnaires completed by the patient, interactions between a 
patient and other school medical offices, issuing alerts immediately after opening the patient's computerized file (if a patient has a critical allergy or if he is undergoing anticoagulant treatment, for example) and also development of phased treatment plans adapted to each patient needs.

Reports: Obtaining reports containing processed and synthesized data, thus providing critical information in the process of developing medium and long-term strategies.

Notifications: Establishing clear tasks for the entire team (doctors, nurses, coordinators) and monitoring their fulfillment (addition of manual or automatic notifications for any activity: periodic check-up, post-intervention check-up, patient birthdays, equipment maintenance, etc.)

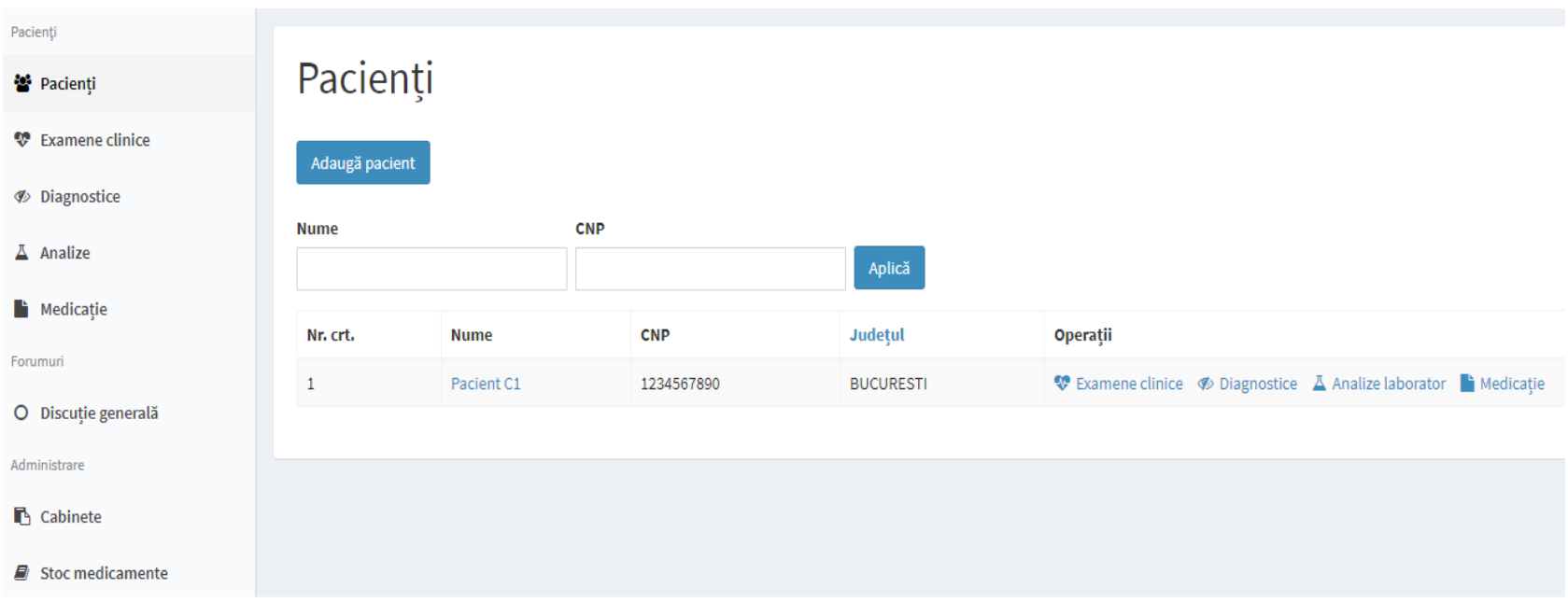

Figure 2. Medical software Medschool print screen from „Patients Page” Source: Medical Software Medschool

Stocks: Evidence of the stock of medicines, sanitary materials, fixed assets and consumables allocated per office. The need to constantly know the stock at the level of medical offices is essential in the proper conduct of medical activity, so measures can be taken to ensure the permanence of the stock at the level of each office. Another important feature of this functionality is the economic part, in this sense, based on the consumption in each office, the materials used in each office can be downloaded from the accounting.

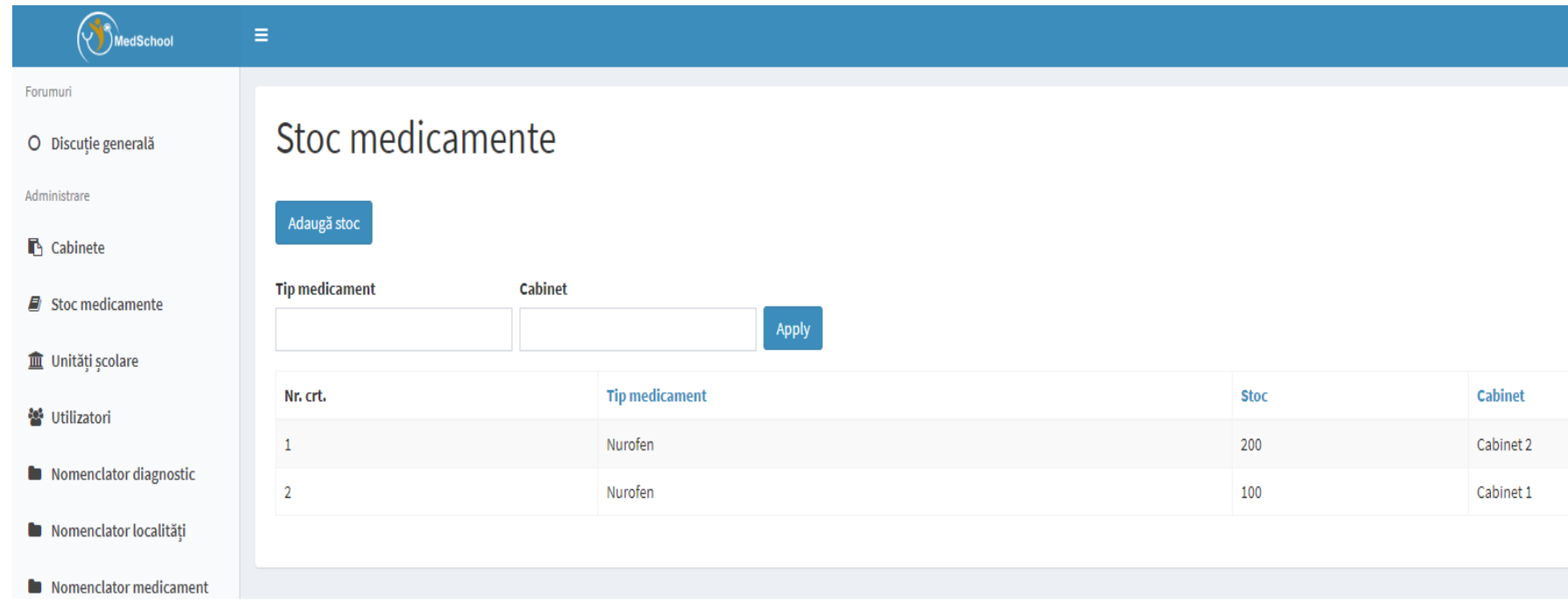

Figure 3. Medical software Medschool print screen from „Stock of medicines and sanitary materials"

Source: Medical Software Medschool 
Settings: Full control over the application, by an administrator, users and all data in the system, which involves managing in the simplest way all important aspects: users, passwords, access rights in the application, list of services, institution data / cabinet, SMS models, etc.

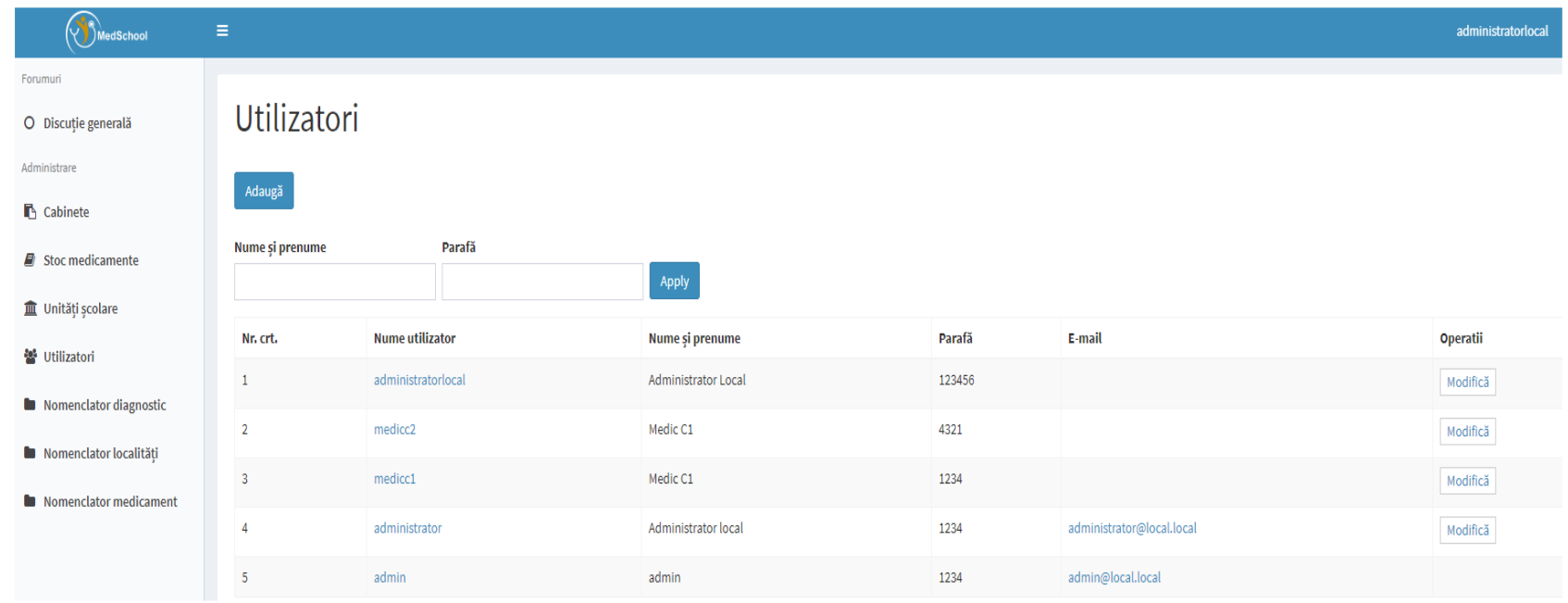

Figure 4. Medical software Medschool print screen from "Stock of users interface" Source: Medical Software Medschool

Automatic backup: Data security, through regular cloud backups at the highest level of data security and operational reliability, using the most reliable Disaster Recovery solutions.

\subsection{Advantages of using software in school medicines in Bucharest}

The advantages of implementing this large-scale digital project, unique in the country, cover the needs that have been resulted from the research process:

1. Keeping and accessing patient records, medical forms and any other medical documents and information about patients;

2. Instant access to the patient's file, with all relevant medical details;

3. Recording, storing and organizing information in one place;

4. Quick access to each patient's history;

5. Automatic generation of mandatory forms and questionnaires for each patient;

6. Maximum security for patient data.

\subsection{Protection of personal data}

Another result derived from the research process was the need to identify all national and international legislation in order to comply with the issue of the protection of personal data, in addition, medical records are considered sensitive information on data.

The processing of data on the medical history of students can only be done with the written consent of the parent or legal guardian.

The parent or legal guardian must complete the agreement by which he accepts or does not accept the data processing, a document that he receives from the educational unit in the first week from the beginning of the school year. The agreement can also be accessed online, on the official website of the A.S.S.M.B., completed and then sent to the school where the pupils attends the courses.

The purpose of processing people's data is to enter the data from the child's / student's medical file which already exists in physical format at the school medical offices - as well as the data related to health care, in an electronic database of general and dental offices. within the state education units from Bucharest. 
In the process of processing personal data, A.S.S.M.B. has complied and will continue to comply, in the future, strictly and fully, with all relevant regulations in national and European legislation.

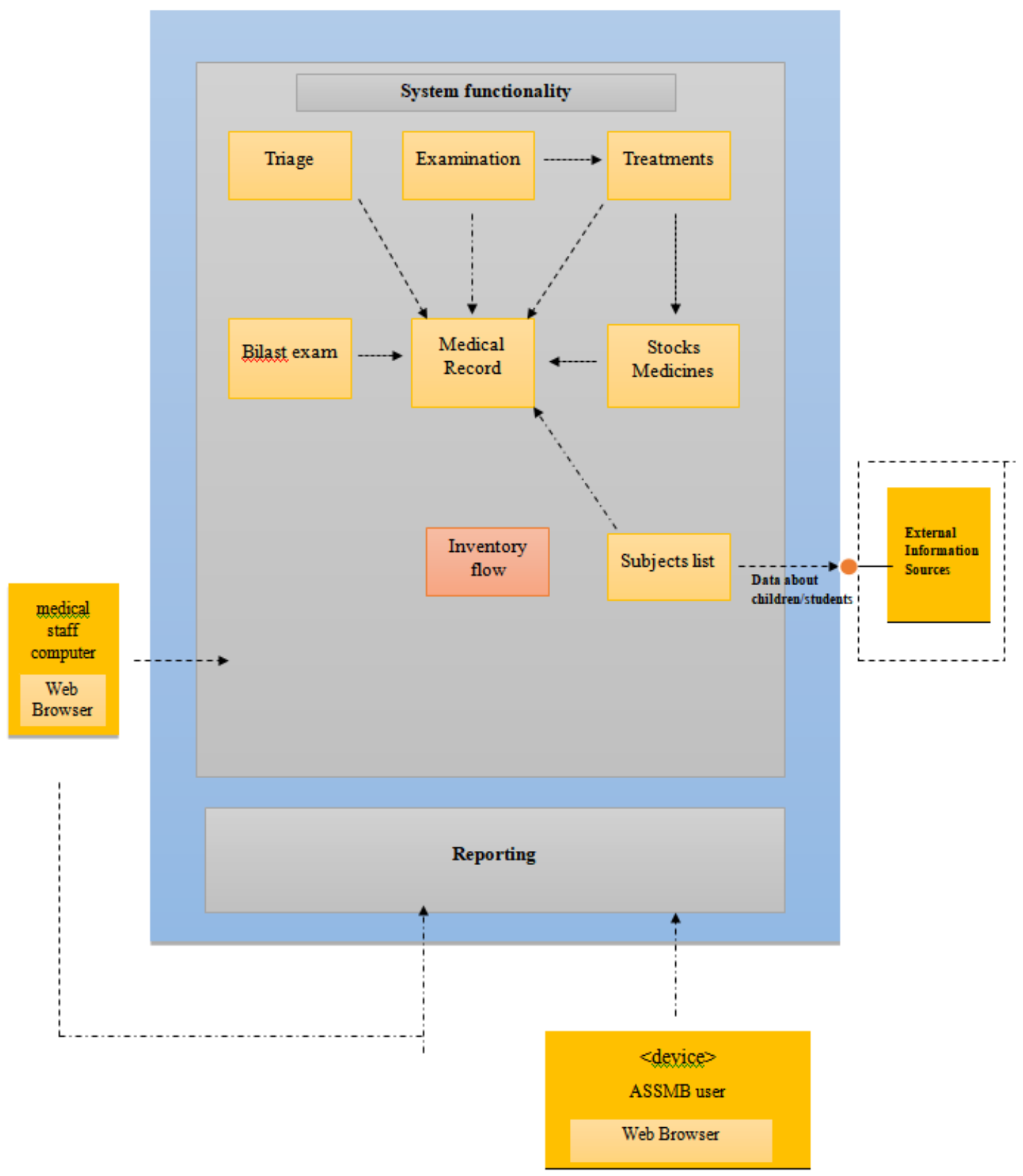

Figure 5. - Logical architecture of the Medschool software digitization Source: Created by the author

\section{CONCLUSIONS}

Amid COVID-19, taking decisive action becomes an imperative. Against the backdrop of high demand for unemployment and other benefits, governments need to ensure speedy and efficient processes and compressed delivery timelines - particularly to alleviate backlogs that have built up during the pandemic. (Matthias Daub, Axel Domeyer, Abdulkader Lamaa, Frauke Renz, 2020).

Thus, related to the research method that has led to the finance problem, the current pandemic situation as well as to the importance and necessity of the digitalization of public institutions and of 
the sanitary field, the school medicine offices have represented a challenge for the management in the digital era.

A.S.S.M.B. has taken the steps to modernization and digitalization of the activity of the school medical offices, considered always, essentially and primarily the interest of ensuring a medical assistance of the highest quality level for the preschoolers, pupils and students of the state education.

In order to achieve this goal, the A.S.S.M.B. has constantly benefited from the support of the School Inspectorate of Bucharest (I.S.M.B.), a collaboration protocol being signed, in this regard between the two institutions, and also the support of the National Federation of Parents' Associations - Bucharest, an organization with which A.S.S.M.B. has a partnership agreement.

Also, the digitization of registers will create a database with a lot of data (Big data) that can be used by the authorities to monitor the health of young citizens, so that resources are directed where there is the biggest need for intervention.

The digitization process is a complex and long one, with direct implications on all beneficiaries, in this case with benefits both among students and parents and among medical staff.

The steps and efforts to implement this digitization process were essential and involved financial, human and time-consuming resources, taking into account factors such as: reluctance of medical staff to change, the legal framework applicable to school medicine practices, data protection legislation and personal character, confidentiality of information, etc.

But the evolution of digitalization is rapid, and the pandemic generated by the SARS Cov-2 virus has shown us its importance and necessity in the medical field.

\section{REFERENCES}

Order issued by the Ministry of Health and the Ministry of Education and Research, no. 438 / 4.629 / 2021 on ensuring the medical assistance of preschoolers, pupils in pre-university education units and students in higher education institutions for maintaining the health of communities and for promoting a healthy lifestyle.

Plesner, U., Justesen, L., \& Glerup, C. (2018). The Transformation of Work in Digitized Public Sector Organizations. Journal of Organizational Change Management, 31(5), 1176-1190. DOI: 10.1108/JOCM-06-2017-0257

Emergency Ordinance no. 38/2020 refers to documents in electronic form at the level of public authorities and institutions

Regulation (EU) 2016/679 of the European Parliament and of the Council of 27 April 2016 on the protection of natural persons with regard to the processing of personal data and on the free movement of such data, and repealing Directive 95/46/EC (General Data Protection Regulation)

https://www.mckinsey.com/industries/public-and-social-sector/our-insights/digital-public-services-howto-achieve-fast-transformation-at-scale (Retrieved September $15^{\text {th }}$, 2021)

https://rohealthreview.ro/assmb-digitalizarea-retelei-de-medicina-scolara-din-bucuresti-acopera-toatecele-609-cabinete-medicale-din-unitatile-de-invatamant/ (Retrieved September 15 ${ }^{\text {th }}$, 2021)

https://www.edupedu.ro/reteaua-de-medicina-scolara-din-bucuresti-are-de-azi-un-program-prin-caredatele-medicale-ale-elevilor-sunt-informatizate-pentru-stocarea-lor-e-nevoie-de-acordulparintilor/ (Retrieved September 15 $5^{\text {th }}, 2021$ )

https://rohealthreview.ro/rapoartele-medicale-ale-elevilor-pot-fi-trimise-direct-catre-parinti-prin-

digitalizarea-cabinetelor-scolare-din-bucuresti/ (Retrieved September 15 th , 2021)

https://assmb.ro/page/baza-legal (Retrieved September 15 ${ }^{\text {th }}, 2021$ )

https://romania.europalibera.org/a/pnrr-reforme-obligatorii-romania/31480457.html (Retrieved September 15 $5^{\text {th }}$, 2021)

https://mfe.gov.ro/wp-content/uploads/2020/07/5e0bdcbddccca4d66d74ba8c1cee1a68.pdf (Retrieved September $15^{\text {th }}$, 2021) 